\title{
BMJ Open Discrepancy between subjectively reported symptoms and objectively measured clinical findings in dry eye: a population based analysis
}

\author{
Rui Hua, ${ }^{1}$ Kai Yao, ${ }^{2}$ Yuedong Hu, ${ }^{1}$ Lei Chen ${ }^{1}$
}

To cite: Hua R, Yao K, Hu Y, et al. Discrepancy between subjectively reported symptoms and objectively measured clinical findings in dry eye: a population based analysis. BMJ Open 2014;4: e005296. doi:10.1136/ bmjopen-2014-005296

- Prepublication history for this paper is available online. To view these files please visit the journal online (http://dx.doi.org/10.1136/ bmjopen-2014-005296).

Received 19 March 2014 Revised 29 July 2014 Accepted 7 August 2014

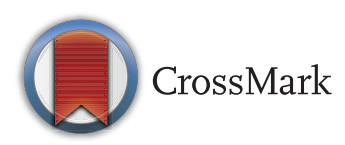

${ }^{1}$ Department of Ophthalmology, First Hospital of China Medical University, Shenyang, China

${ }^{2}$ Department of

Ophthalmology and Visual Science, Yale University School of Medicine, New Haven, Connecticut, USA

Correspondence to Professor Lei Chen; LeiChen51@126.com

\section{ABSTRACT}

Objective: To investigate the discrepancy between patient-reported symptoms and measured clinical findings and influencing factors in dry eye (DE).

Setting: A population-based, cross-sectional study was performed in July-August 2007 in northeast China. The study was performed on populations originating from two rural districts that are respectively located approximately 377 and $177 \mathrm{~km}$ from our hospital.

Participants: 2600 eligible residents from 1300 households were identified; valid responses were obtained from 2262 residents (mean age 48 (range 12-88) years; 926 men and 1336 women; response rate $87 \%$ ).

Primary outcome measures: Patient-reported symptoms of DE, tear film break up time (BUT) and Schirmer scores (Schirmer II).

Results: Subjects with normal BUT and Schirmer scores without any DE symptoms were defined as the control group. Those with abnormal BUT and Schirmer scores and symptoms of DE were defined as the $D E$ group. Subjects with disparities between the occurrence of DE symptoms and measured clinical findings were regarded as the discrepancy. Out of 2262 subjects, the discrepant group contained 960 subjects (42.44\%) with significant difference $\left(\chi^{2}=4.027, p=0.045<0.05\right)$. Factors that influenced the subjective reporting of $D E$ symptoms included gender, smoking status, environment and age. Schirmer II test and tear film BUT values were remarkably different among control, DE and discrepant groups.

Conclusions: Development of DE can be related to many factors. It is of great importance to put forward the preclinical phase concept (patients who are symptomatic for $D E$ and yet show no aqueous deficiency or evaporative signs) and to screen outpatients with $\mathrm{DE}$-inducing features. Future interventions should focus on patients demonstrating a discrepancy between self-reported symptomology and measured clinical findings.

\section{INTRODUCTION}

Dry eye (DE) is a disorder affecting the tear ducts and ocular surface, which can be caused by many factors, and which produces

\section{Strengths and limitations of this study}

- To our knowledge, this is the first report describing the discrepancy between subjective symptoms and objectively measured clinical findings in dry eye in a large Chinese patient sample.

- Large-scale, population-based dry eye epidemiologic studies on this discrepancy have been limited in China.

- Our study supports results produced by the Diagnostic Methodology Subcommittee of the International Dry Eye Workshop, which demonstrated that the administration of a structured questionnaire to patients at the time of presentation to the clinic provides an excellent opportunity to screen for patients suffering from potential dry eye disease.

- Tear osmotic pressure (tear osmolarity) was not measured in our diagnostic protocol.

- Our protocol also excluded some of the other known clinical tests for evaluating the ocular surface.

symptoms including ocular discomfort, visual disturbances and tear film instability. DE can also lead to potential damage of the ocular surface and is accompanied by both increased tear film osmolarity and inflammation of the ocular surface. ${ }^{1}$ Despite these considerations, the diagnosis of DE is frequently overlooked in the clinic and has not yet been widely accepted in China. When clinically identifying $\mathrm{DE}$, in addition to primary auxiliary examinations, including measurement of visual acuity, external examination and slit-lamp biomicroscopy, ${ }^{2}$ further diagnostic tests should be performed to evaluate the extent of ocular surface damage (eg, staining with rose bengal, lissamine green or fluorescein dye), tear film instability (eg, tear break up time (BUT) test) and the aqueous tear flow (eg, Schirmer test) ${ }^{3}$ of a given patient. It has recently been reported that DE symptoms, as evaluated subjectively 
in patient questionnaires, occur in approximately $21 \%$ of the adult population within China. Furthermore, it was found that psychological depression is associated with $\mathrm{DE}$, particularly in aging patient populations from Beijing. ${ }^{4}$ Although population-based survey studies of DE have been performed globally, ${ }^{6} 7$ the nature of this disorder has not been well documented within Chinese populations. We performed the present survey to examine the lack of correlation between the subjective presentation of DE symptoms, and two objective clinical tests of DE, within selected Chinese patient groups. Additionally, we analysed the correlation of smoking and environmental humidity to the incidence of DE.

\section{METHODS}

A population-based, cross-sectional study was performed on patients living in northeast China from July to August, 2007. The study was carried out in two rural districts, Zhuanghe and Dawa, which are located approximately 377 and $177 \mathrm{~km}$ from our hospital, respectively. The Zhuanghe district is located near the Bohai Sea, while the Dawa district is inland. The majority of inhabitants from either district work as farmers. All of the people living in these communities were officially registered by name, gender and age through the local government, ensuring that the boundaries and population density of each community were known at the beginning of the study. Home visits were performed according to these registration lists. All of the enrolled subjects were aged 12 and over and were selected via cluster sampling. Subject choices were confirmed by door-to-door visitation. Residents were deemed ineligible and excluded from study for reasons including death, moving out of town, nursing or hospitalisation. A total of 2600 eligible residents from 1300 households were identified, and valid responses were obtained from 2262 residents (mean age 48 (range 12-88) years; 926 men and 1336 women; response rate $87 \%$ ). If a subject was unwilling to join the study, or did not receive all the tests outlined in our diagnostic protocol, an invalid response was recorded. Subjects with a documented history of ocular surface disease, such as keratitis or conjunctivitis, were excluded. Subjects were also excluded if such lesions were detected via slit lamp (Su Zhou SIX-SIX Technological Development Co., China) during initial visitation. After answering a self-administered questionnaire distributed by the investigators, all of the eligible subjects from the same community were then brought to a central location for clinical investigation. All the experiments and measurements adhered to the ethical principles of the Declaration of Helsinki and were approved by the Medical Research Ethics Committee of the First Hospital of China Medical University. Written informed consent was obtained from all participants.

All subjects were first required to complete a questionnaire survey about epidemiological investigation (the

\section{Box 1 Dry eye questionnaire used in this survey}

1. Do your eyes ever feel dry?

2. Do your eyes ever have tearing?

3. Do your eyes ever feel burning?

4. Do your eyes ever have blurring and fluctuating vision?

5. Do your eyes ever feel irritation?

6. Do your eyes ever feel foreign body sensation?

7. Do your eyes ever feel tired?

Possible answers to the questions were 'none or rarely', 'sometimes' and 'often or all the time'. Positive symptoms were defined by the answers 'sometimes' or 'often or all the time'.

clinic); following this, their tear secretion volumes and tear film BUT values were recorded.

We evaluated DE symptoms using the seven questions developed and validated by Gulati et $a l^{4}{ }^{89}$ which focus on ocular discomforts: awareness, tearing, burning, blurring and fluctuating vision, irritation, foreign body sensation, and tired eyes (box 1). Those subjects who identified a minimum of three out of seven questions as positive were considered 'symptom positive'. Smoking status was also recorded. The above data were jointly recorded by two investigators (RH, YDH) during in-home interviews of prospective subjects.

\section{BUT}

To avoid any interference, the BUT test was performed prior to other DE tests. Fluorescein filter paper (Tianjin Jingming New Technological Development Co., China) was moisturised and placed on the eye to coat the lower temporal side of the bulbar conjunctiva. The subjects were advised to blink several times to ensure that the corneal surface became evenly coated with $2 \%$ fluorescein. Tear film stability was measured using a slit lamp equipped with a cobalt blue filter to record the time elapsed from the last complete blink to the appearance of the first random dry spot. The slit lamp and filter were standardised across the studies.

\section{Schirmer test}

To avoid ocular irritation caused by the test strip from interfering with other examinations, the Schirmer test was the final test performed during patient evaluation. Tear secretion test filter paper (Tianjin Jingming New Technological Development Co.) measuring $35 \mathrm{~mm}$ in length, with a bend at $5 \mathrm{~mm}$, was used. At $1 \mathrm{~min}$ following the application of topical anaesthesia $(20 \mathrm{~mL} ; 80 \mathrm{mg}$ oxybuprocaine hydrochloride eye drops, Santan, Japan), the filter paper was placed at the junction of the medial two-thirds and lateral one-third of the lower lid in the fornix. In addition, the test was carried out under dim illumination and standardised conditions of temperature and humidity (temperature and humidity were maintained at $20-25^{\circ} \mathrm{C}$ and $45-65 \%$, respectively). The amount of moisture present on the filter paper was recorded at $5 \mathrm{~min}$ after paper application. Afterwards, 
the subjects were asked to blink normally. Positive clinical findings were defined as a patient having both a Schirmer II test result of less than $10 \mathrm{~mm}$ per $5 \mathrm{~min}$ and a tear film BUT value of less than $10 \mathrm{~s} .{ }^{10}$ Subjects with normal BUT and Schirmer scores, and without any reported symptoms of DE, were defined as the control group. Subjects with abnormal BUT and Schirmer scores, as well as reported symptoms of DE, were defined as the DE group. Finally, subjects with inconsistencies between symptom occurrence and clinical findings were regarded as the discrepancy.

\section{Statistical analysis}

All data were analysed using SPSS (V.19.0) and expressed as median values (min-max). Regression analysis was performed to adjust for gender, age, smoking status and geographical location. The Pearson $\chi^{2}$ test was used to assess the proportional values between the two cohorts, as well as the influencing factors (gender and environment) in subjects with positive clinical findings. The influencing factors (age) in those subjects, and the differences in Schirmer I test and BUT values were analysed using the Kruskal-Wallis $\mathrm{H}$ test. $\mathrm{p}$ Values of less than 0.05 were considered statistically significant.

\section{RESULTS}

Of the 2262 subjects studied, 1710 subjects presented with symptoms of DE and 1354 subjects had low BUT and Schirmer values. Additionally, the discrepant group contained $960(302+658,42.44 \%)$ subjects, which is statistically significant (Pearson $\chi^{2}$ test: $\chi^{2}=4.027$, $\mathrm{p}=0.045<0.05$; table 1 ). Of the 1302 subjects demonstrating consistency between reported symptoms and measured clinical findings, 1052 were in the DE group, and the remainder in the control group. The sensitivity and specificity of DE identification based on subject symptoms were $77.70 \%(1052 / 1354)$ and $27.53 \%$ (250/908), respectively, while the accuracy of using the subjects' perceived symptoms for DE identification was $57.56 \%$ $((1052+250) / 2262)$. Additionally, the positive predictive

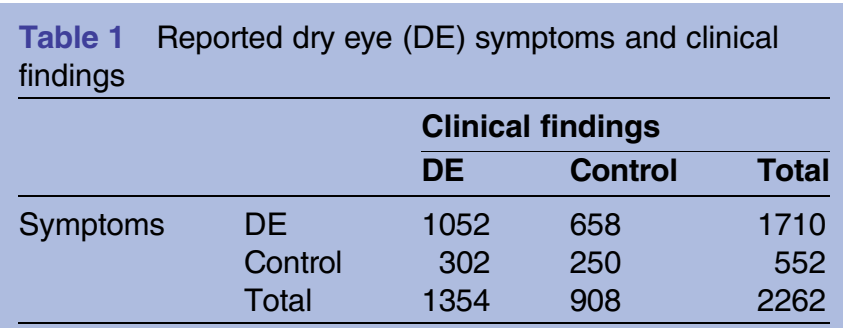

The discrepant group contained $960(302+658,42.44 \%)$ subjects, which is significant in statistics (Pearson chi-square test: $\chi^{2}=4.027$, $\mathrm{p}=0.045<0.05$ ). The sensitivity and specificity of DE identification based on subject symptoms were $77.70 \%(1052 / 1354)$ and $27.53 \%(250 / 908)$, respectively, and the accuracy of using the subjects' perceived symptoms for DE identification was $57.56 \%$ $((1052+250) / 2262)$. The positive predictive value was $61.52 \%$ (1052/1710), and the negative predictive value was $45.29 \%$ (250/552). value and likelihood ratios were $61.52 \%$ (1052/1710) and $1.072(77.70 \% /(1-27.53 \%))$, respectively, while the negative predictive value and likelihood ratios were $45.29 \%(250 / 552)$ and $0.810 \quad((1-77.70 \%) / 27.53 \%)$, respectively.

Logistic analysis showed that there was no relationship between symptom presentation and clinical findings in this study $(\mathrm{OR}=1.112, \mathrm{p}=0.495>0.05)$. Moreover, gender $(\mathrm{OR}=2.059, \quad \mathrm{p}<0.0001)$, smoking status $(\mathrm{OR}=2.263$, $\mathrm{p}<0.0001$ ) and geographical region (coastal region or inland region; $\mathrm{OR}=0.272, \mathrm{p}<0.0001$ ) were risk factors for subjectively reported $\mathrm{DE}$ symptoms, rather than age $(\mathrm{OR}=1.400, \mathrm{p}=0.100>0.05)$.

Of the 1354 subjects with positive clinical findings, 622 of $780(87.12 \%)$ female subjects presented with related symptoms, while 390 of $574(89.51 \%)$ men presented with related symptoms. Women were more likely than men to experience symptoms of DE $\left(\chi^{2}=12.193\right.$, $\mathrm{p}<0.0001)$. With regard to patients living in the coastal region, 574 of $820(70.00 \%)$ subjects presented with $\mathrm{DE}$ symptomology, while the percentage of symptomatic patients living inland was $89.51 \%$ (478 of 534 subjects). Thus, subjects living inland made up a higher proportion than those living at the coast $\left(\chi^{2}=35.528, p<0.0001\right)$. Furthermore, significant differences in whether subjects presented with DE symptomology were found to correlate with patient age $(\mathrm{Z}=1.983, \mathrm{p}=0.047<0.05)$.

There were remarkable differences in the values obtained for Schirmer II testing and tear film BUT among our three study groups (Schirmer II test: $\chi^{2}=422.93, \mathrm{p}<0.0001 ;$ BUT: $\chi^{2}=264.85, \mathrm{p}<0.0001$; table 2 ).

\section{DISCUSSION}

DE or dysfunctional tear syndrome is one of the most frequent diagnoses in ophthalmology. Risk factors for the syndrome include age, sex (female gender), race, use of contact lenses, environmental conditions of low humidity, systemic medications and autoimmune disorders. ${ }^{11}$

Tear film components contain meibomian lipids, ocular mucins and proteins. Tear film stability can be assessed via a number of approaches developed for clinical as well as research purposes, including evaluation of

Table 2 Primary outcome variables of tear film break up time (BUT) and Schirmer scores (Schirmer II) in the subject groups

\begin{tabular}{lllr}
\hline Symptoms & $\begin{array}{l}\text { Clinical } \\
\text { findings }\end{array}$ & $\begin{array}{l}\text { Schirmer II } \\
\text { test }(\mathbf{m m})\end{array}$ & \multicolumn{1}{c}{ BUT (s) } \\
\hline Normal & Normal & $22.0(2.0-30.0)$ & $12.5(0.0-30.0)$ \\
Discrepancy & & $15.0(0.0-30.0)$ & $9.0(0.0-30.0)$ \\
DE & DE & $5.0(0.0-30.0)$ & $4.0(0.0-20.0)$ \\
\hline
\end{tabular}

There were remarkable differences in the values obtained for Schirmer II testing and tear film BUT among our three study groups (Schirmer II test: $\chi^{2}=422.93$, p<0.0001; BUT: $\chi^{2}=264.85$, $\mathrm{p}<0.0001)$.

$\mathrm{DE}$, dry eye. 
tear BUT and non-invasive break time, topographic and interferometric techniques, confocal microscopic methods, aberrometry and visual function tests. ${ }^{12}$ In the present study, Schirmer II testing and BUT were performed. It is widely accepted that measurements of tear hyperosmolarity are the 'gold standard' for diagnosing $\mathrm{DE},{ }^{2}$ as DE leads to cell apoptosis in the conjunctiva and cornea and triggers inflammatory cascades that contribute to further cell death, including the loss of mucinproducing goblet cells, which exacerbates tear film instability. ${ }^{13}$ However, such approaches are difficult to perform under the conditions of epidemiological investigation. BUT is also considered a top choice in clinical testing, as it also measures the mechanisms discussed above with good overall accuracy, ${ }^{14}$ and appears to be more repeatable across patient visits than many other diagnostic tests. ${ }^{15}$ As a result, BUT is a more suitable choice for epidemiological investigation.

The number of patients in the discrepant group, those who did not report DE symptomology, was $42.44 \%$. With the positive and negative likelihood ratio values both being close to 1 (1.072 and 0.810 , respectively), it was found that the possibility of correctly identifying DE based on symptomology alone is equivalent to the possibility of incorrectly identifying it.

Similarly, Schein ${ }^{16}$ concluded that there are distinct differences between the chief symptomatic complaints of patients and lab results, making it difficult to distinguish such lesions. To our knowledge, there are several reasons behind the disparities found between subjective self-reporting and the measured clinical findings that are vital to performing routine clinical work.

First, there are gender differences. Chia et al reported that women have a higher tendency to develop symptomatic DE. This finding may be associated with genderrelated hormone levels, ${ }^{17}$ as the androgen pool of non-autoimmune DE patients with meibomian gland dysfunction (MGD) is significantly depleted compared with that of non-MGD and control cases. ${ }^{18}$ Our findings agree with the Chia study that female gender is a risk factor for DE.

Second, environmental conditions play an important role in the development of DE. For example, locally elevated evaporation rates lead to hyperosmolar spots within the tear film and subsequent vulnerability to epithelial irritation. In addition to evaporation rates, tear film instability depends on the strength of healing flow from the neighbouring regions of the eye that lie outside the breakup region. ${ }^{19}$ Many DE patients are sensitive to adverse environments, where tear evaporation rates (TER) increase due to a reverse correlation when environmental humidity is in the range $5-70 \%$. In fact, TER is reduced to zero at $70 \%$ relative humidity. ${ }^{20}$ Additionally, adult patients exhibiting mild-to-moderate $\mathrm{DE}$ and asymptomatic subjects of similar ages can both experience acute exacerbation of the disorder following exposure to a controlled desiccating environment $(5 \%$ relative humidity) for $2 \mathrm{~h}^{21}$ Dawa, being an inland location, generally has lower atmospheric humidity which leads to distinctive DE symptomology, as increasing periocular humidity has been demonstrated to have a significant positive impact on ocular comfort in DE patients. ${ }^{22}$ Additionally, Sayin $e t a l^{23}$ reported that cigarette smoking appears to affect Schirmer score values, tear BUT values and the hexagonal cells of the corneal endothelium, which further supports our results.

The lack of correlation between objective clinical findings and subjective symptomatic reporting is not uncommon. For example, early detection of glaucoma is often difficult as it is frequently asymptomatic during the initial stages of the disease. Studies have shown that the majority of glaucoma cases are not diagnosed until later stage disease progression has occurred. ${ }^{24}$ Uchino et al reported short BUT and corneal staining accompanied by normal Schirmer test values, ${ }^{25}$ while we found a discrepancy in DE between subjective symptom reporting and measured clinical findings with regard to BUT and Schirmer test values. Similarly, no consistent relationship was found between self-reported symptoms of DE and objectively measured clinical findings in the EU and USA. As symptomology alone is insufficient for the diagnosis and management of $\mathrm{DE}$, it is arguable that a consensus of clinical signs is needed to better reflect all aspects of the disease. ${ }^{26}$ Thus, a combined test and set criteria for diagnosis and differentiation of $\mathrm{DE}$ are important for improving future DE research. Additionally, in subjects producing positive clinical findings, symptomatic complaints of DE were accompanied by reduced tear secretion volumes and lower tear film stability values, which suggests the existence of a 'latent stage' in DE. Sullivan et $a l^{27}$ also reported that the initiation and progression of $\mathrm{DE}$ is multifactorial, which further supports the rationale for redefining DE severity on the basis of a continuum of clinical symptoms. In this study, we ranked all subjects according to the severity of the reduction in tear secretion and tear film BUT values. Subjects were classified as: (1) having no presentation of symptoms or measured clinical findings; (2) having disparity between DE symptom presentation and positive or negative measured clinical findings; and (3) symptomatic of DE with positive measured clinical findings.

Future longitudinal studies will be necessary to follow $\mathrm{DE}$ lesion progression in asymptomatic subjects. Furthermore, more attention needs to be devoted towards following subjects that present with a discrepancy between symptomatic reporting and measured clinical findings-a group which has historically been disregarded in DE research.

It was widely accepted that increasing subject age is closely related to the severity of DE symptomology. It has recently been reported that DE is prevalent among young to middle-aged Japanese subjects who use visual display terminals. An increased DE risk was also noted in women aged over 30 years. ${ }^{25}$ In this survey, age was not found to be a risk factor for symptomatic DE across all 
subjects; however, in subjects with positive clinical findings, we found that subject age did correlate significantly with whether there was DE symptomology. We suggest that this may be influenced by the inclusion of the discrepant group in our analyses.

Our study included some inherent limitations. For example, we did not test tear osmotic pressure in our diagnostic protocol, and did not directly evaluate the ocular surface or the meibomian glands. Our protocol also lacked some of the additional objective tests that can be used to evaluate the ocular surface. These limitations will be addressed in future studies.

Our findings suggest that there are many subjects that potentially suffer from DE despite a lack of reported symptomology. The population examined in this study was comprised mainly of farmers, which suggests that more attention should be paid to this special group. Additionally, it is necessary to screen those outpatients possessing DE inducing factors, and future interventions should focus on patients demonstrating discrepancies between symptomology and measured clinical findings. Similarly, the Diagnostic Methodology Subcommittee of the International Dry Eye Workshop also concluded that the administration of a structured questionnaire to patients presenting to the clinic provides an excellent opportunity for screening patients with potential DE disease. ${ }^{14}$

In conclusion, the causes of DE are multifactorial; factors that influence the severity of DE symptomology include gender, smoking, environment and age. Moving forward, it is of great importance to make the progression of DE clear, to put forward the preclinical phase concept and to recognise the discrepancies found in many subjects, all of which may contribute favourably to the prevention, diagnosis and treatment of DE.

Acknowledgements This work was supported in part by Dr Lei Liu, Dr Yizhou Sun and Dr Jun Chen of the Department of Ophthalmology, First Hospital of China Medical University. We also thank the American Journal Experts (AJE) for assisting in the preparation of this manuscript.

Contributors Study concept and design: LC and RH. Acquisition of data: RH and YDH. Analysis and interpretation of data: RH, YDH and LC. Drafting of the manuscript: $\mathrm{RH}$ and $\mathrm{KY}$. Critical revision of the manuscript for intellectual content: LC. All authors have read and approved the final manuscript.

\section{Competing interests None.}

Patient consent Obtained.

Ethics approval The Medical Research Ethics Committee of First Hospital of China Medical University.

Provenance and peer review Not commissioned; externally peer reviewed.

Data sharing statement No additional data are available.

Open Access This is an Open Access article distributed in accordance with the Creative Commons Attribution Non Commercial (CC BY-NC 4.0) license, which permits others to distribute, remix, adapt, build upon this work noncommercially, and license their derivative works on different terms, provided the original work is properly cited and the use is non-commercial. See: http:// creativecommons.org/licenses/by-nc/4.0/

\section{REFERENCES}

1. Lemp MA, Baudouin C, Baum J, et al. The definition and classification of dry eye disease: report of the definition and classification subcommittee of the international dry eye workshop (2007). Ocul Surf 2007;5:75-92.

2. Bron AJ. Diagnosis of dry eye. Surv Ophthalmol 2001;45(Suppl 2): S221-6.

3. Perry HD. Dry eye disease: pathophysiology, classification, and diagnosis. Am J Manag Care 2008;14:S79-87.

4. Jie $\mathrm{Y}, \mathrm{Xu} \mathrm{L}, \mathrm{Wu} \mathrm{YY}$, et al. Prevalence of dry eye among adult Chinese in the Beijing Eye Study. Eye (Lond) 2009;23:688-93.

5. Labbé A, Wang $Y X$, Jie $Y$, et al. Dry eye disease, dry eye symptoms and depression: the Beijing Eye Study. Br J Ophthalmol 2013:97:1399-403.

6. Onwubiko SN, Eze BI, Udeh NN, et al. Dry eye disease: Prevalence distribution and determinants in a hospital-based population. Cont Lens Anterior Eye 2014;37:157-61.

7. van Landingham SW, West SK, Akpek EK, et al. Impact of dry eye on reading in a population based sample of the elderly: the salisbury eye evaluation. Br J Ophthalmol 2014;98:639-44.

8. Gulati A, Sullivan R, Buring JE, et al. Validation and repeatability of a short questionnaire for dry eye syndrome. Am J Ophthalmol 2006;142:125-31

9. Uchino M, Nishiwaki Y, Michikawa T, et al. Prevalence and risk factors of dry eye disease in Japan: Koumi study. Ophthalmol 2011;118:2361-7

10. Mei Z, Jiaqi C, Zhuguo L. The diagnosis of dye eye syndrome. Chin J Pract Ophthalmol 2000;18:664-8.

11. Kaštelan S, Tomić M, Salopek-Rabatić J, et al. Diagnostic procedures and management of dry eye. Biomed Res Int 2013;2013:309723.

12. Sweeney DF, Millar TJ, Raju SR. Tear film stability: a review. Exp Eye Res 2013;117:28-38.

13. Baudouin C, Aragona P, Messmer EM, et al. Role of hyperosmolarity in the pathogenesis and management of dry eye disease: proceedings of the OCEAN group meeting. Ocul Surf 2013;11:246-58.

14. Bron AJ. Methodologies to diagnose and monitor dry eye disease: report of the diagnostic methodology subcommittee of the International Dry Eye Workshop (2007). Ocul Surf 2007;5:108-52.

15. Nichols KK, Mitchell GL, Zadnik K. The repeatability of clinical measurements of dry eye. Cornea 2004;23:272-85.

16. Schein OD, Tielsch JM, Munõz B, et al. Relation between signs and symptoms of dry eye in the elderly. a population-based perspective. Ophthalmology 1997;104:1395-401.

17. Chia EM, Mitchell $P$, Rochtchina $E$, et al. Prevalence and associations of dry eye syndrome in an older population: the Blue Mountains Eye Study. Clin Experiment Ophthalmol 2003;31:229-32.

18. Tamer $\mathrm{C}$, Oksuz $\mathrm{H}$, Sogut $\mathrm{S}$. Androgen status of the nonautoimmune dry eye subtypes. Ophthalmic Res 2006;38:280-6.

19. Peng CC, Cerretani C, Braun RJ, et al. Evaporation-driven instability of the precorneal tear film. Adv Colloid Interface Sci 2014;206:250-64.

20. Madden LC, Tomlinson A, Simmons PA. Effect of humidity variations in a controlled environment chamber on tear evaporation after dry eye therapy. Eye Contact Lens 2013;39:169-74.

21. López-Miguel A, Tesón M, Martín-Montañez V, et al. Dry eye exacerbation in patients exposed to desiccating stress under controlled environmental conditions. Am J Ophthalmol 2014;157:788-98.

22. Korb DR, Blackie CA. Using goggles to increase periocular humidity and reduce dry eye symptoms. Eye Contact Lens 2013;39:273-6.

23. Sayin N, Kara N, Pekel G, et al. Effects of chronic smoking on central corneal thickness, endothelial cell, and dry eye parameters. Cutan Ocul Toxicol 2013. [Epub ahead of print].

24. Rewri P, Kakkar M. Awareness, knowledge, and practice: a survey of glaucoma in north Indian rural residents. Indian $J$ Ophthalmol 2014:62:482-6.

25. Uchino M, Yokoi N, Uchino Y, et al. Prevalence of dry eye disease and its risk factors in visual display terminal users: the Osaka study. Am J Ophthalmol 2013;156:759-66.

26. Sullivan BD, Crews LA, Messmer EM, et al. Correlations between commonly used objective signs and symptoms for the diagnosis of dry eye disease: clinical implications. Acta Ophthalmol 2014;92:161-6.

27. Sullivan BD, Whitmer D, Nichols KK, et al. An objective approach to dry eye disease severity. Invest Ophthalmol Vis Sci 2010:51:6125-30. 